\title{
ADAPTIVE REGULARIZED ZERO-FORCING BEAMFORMING in MASSIVE MiMO With Multi-ANTENNA Users
}

A PREPRINT

\author{
Evgeny Bobrov ${ }^{1,2}$ \\ eugenbobrov@ya.ru \\ Hao Lu ${ }^{1}$ \\ luhao12@huawei.com
}

\author{
Boris Chinyaev $^{2}$ \\ bchinyaev.msu@gmail.com
}

\author{
Dmitrii Minenkov ${ }^{2,4}$ \\ minenkov.ds@gmail.com
}

\author{
Viktor Kuznetsov ${ }^{1}$ \\ kuznetsov.victor@huawei.com \\ Sergey Troshin ${ }^{3}$ \\ serj.troshin2013@yandex.ru
}

\author{
Daniil Yudakov $^{2}$ \\ d. yudakov43@gmail.com
}

\author{
Danila Zaev ${ }^{1}$ \\ zaev.danila1@huawei.com
}

July 1, 2021

\begin{abstract}
Modern wireless cellular networks use massive multiple-input multiple-output (MIMO) technology. This involves operations with an antenna array at a base station that simultaneously serves multiple mobile devices that also use multiple antennas on their side. For this, various Beamforming and Detection techniques are used, allowing each user to receive the signal intended for him from the base station. There is an important class of linear Precoding called Regularized Zero-Forcing (RZF). In this work, we propose a special kind of regularization matrix with different regularization for different UE (Adaptive RZF), using singular values of multi-antenna users. Proposed algorithm has a simple analytical formula and is provided with theoretical study. We also show the results in comparison with other linear Precoding algorithms on simulations with the Quadriga channel model. The proposed approach leads to a significant increase in quality with the same computation time as in the baseline methods.
\end{abstract}

Keywords: Massive MIMO, MU-Precoding, Beamforming, Regularized Zero Forcing, SVD

\section{Introduction}

In MIMO systems with a large number of antennas, pre-coding is an important part of downlink signal processing, since this procedure can focus the transmission signal energy on smaller areas and allows for greater spectral efficiency with less transmitted power [1, 2]. Various linear Precodings allow to direct the maximum amount of energy to the user (MRT) or completely get rid of inter-user interference (ZF) [15]. There are also different non-linear Precoding techniques such as dirty paper coding (DPC) and vector perturbation (VP) but they have much higher implementation complexity [4] and in the case of the usage of a large number of antennas in massive MIMO, linear Precoding techniques are more preferable. In the case of RZF, we balance between maximizing the signal power and minimizing the interference leakage [18] and still having low complexity compared to non-linear Precoding.

There are many good surveys of Precoding techniques [6, 5] and interesting works that review many different aspects and variations of these algorithms. In particular, the work [7] presents various variants of RZF in the case of multiple

\footnotetext{
${ }^{1}$ Huawei Russian Research Institute

${ }^{2}$ M. V. Lomonosov Moscow State University

${ }^{3}$ National Research University Higher School of Economics

${ }^{4}$ Ishlinsky Institute for Problems in Mechanics of the Russian Academy of Sciences (IPMech RAS)
} 
base stations, which help to reduce inter-cell interference. However, most works do not pay attention to multi-antenna user equipment (UE) for simplicity. In our work, we consider a single base station serving multi-antenna users who simultaneously receive fewer data channels than their number of antennas. This approach is necessary because, in practice, the channels between different antennas of one UE are often spatial correlated [8]. Therefore, the matrix of the user channel is ill-conditioned (or even has incomplete rank), thus one can not efficiently transmit data using the maximum number of streams.

To solve this problem, instead of the full matrix of the user channel, vectors from its singular value decomposition (SVD) with the largest singular values are used for Precoding [14]. In the case of UE with one antenna the channel matrix can be normalized [16] and normalization coefficients are the path losses of each UE that can differ by several orders (common RSRP values vary from $-70 \mathrm{dBm}$ to $-130 \mathrm{dBm}$ ). When we use SVD this path loss information is put to singular values that therefore are quite different for different UE. Motivated by the above issue, we investigate RZF Precoding in the multi-antenna users' case and propose a simple sub-optimal analytical modification of this algorithm, taking into account the singular values of transmitted layers (streams).

The rest of this paper is organized as follows. In Section 2 we formulate the problem, that consists of Channel and System Model, quality measures and power constraints. The downlink MIMO channel model is simplified using SVD-decomposition of the channel (sec.2.1) and useful idealistic Detection (sec.2.2); quality measures and power constraints are discussed in sec. 2.3, 2.4. In Section 3 we propose an adaptive Precoding algorithm that utilizes UE singular values and study its relation with known Precoding, including MRT, ZF and RZF. Comparison of these algorithms on numerical experiments with quadrigo are provided in Section 4 . Conclusions are drawn in Section 5 .

Throughout the paper we use the following notations. We consider one cell with $K \mathrm{UE}$, the number of transmit antennas is $T=64$ and the number of receive antennas and transmit symbols of UE $k$ are $R_{k}=1,2,4$ and $L_{k} \leq R_{k}$ with total $R=\sum_{k} R_{k}$ and $L=\sum_{k} L_{k}$. By bold lower case letters we denote vector-columns and by bold upper case letters we denote matrices, considering them as sets of vector-columns (e.g. $\boldsymbol{A}=\left(\boldsymbol{a}_{1}, \ldots, \boldsymbol{a}_{R_{k}}\right)$ ). Hermitian conjugate is denoted by $\boldsymbol{A}^{H} \equiv \overline{\boldsymbol{A}}^{T}$. Diagonal and block-diagonal matrices are written as $\boldsymbol{S}_{k}=\operatorname{diag}\left\{s_{k, 1}, \ldots, s_{k, R_{k}}\right\}$ and $\boldsymbol{S}=\operatorname{bdiag}\left\{\boldsymbol{S}_{1}, \ldots, \boldsymbol{S}_{k}\right\}$ correspondingly, the identity matrix of size $M$ is $\boldsymbol{I}_{M}=\operatorname{diag}\{1, \ldots, 1\} \in \mathbb{C}^{M \times M}$. Generally, we use the Frobenius norm $\|\boldsymbol{A}\|=\sqrt{\sum_{i, j}\left|a_{i j}\right|^{2}}$, other options will be discussed below.

\section{Channel and System Model}

According to [2, 11, 9, 10, 12] we consider a MIMO broadcast channel. The Multi-User MIMO model is described using the following linear system:

$$
\boldsymbol{r}=\boldsymbol{G}(\boldsymbol{H} \boldsymbol{W} \boldsymbol{x}+\boldsymbol{n})
$$

Where $\boldsymbol{r} \in \mathbb{C}^{L}$ is a received vector, and $\boldsymbol{x} \in \mathbb{C}^{L}$ is a sent vector, and $\boldsymbol{H} \in \mathbb{C}^{R \times T}$ is a downlink channel matrix, and $\boldsymbol{W} \in \mathbb{C}^{T \times L}$ is a Precoding matrix, and $\boldsymbol{G} \in \mathbb{C}^{L \times R}$ is a block-diagonal Detection matrix, and $\boldsymbol{n} \sim \mathcal{C N}\left(0, \sigma^{2} I_{L}\right)$ is a noise-vector and $\sigma^{2}$ is noise level. Note that the linear Precoding and decoding are implemented by simple matrix multiplications. The constant $T$ is the number of transmit antennas, $R$ is the total number of receive antennas, and $L$ is the total number of transmitted symbols in the system. Usually they are related as $L \leqslant R \leqslant T$. Each of the matrices $\boldsymbol{G}, \boldsymbol{H}, \boldsymbol{W}$ decomposes by $K$ users: $\boldsymbol{G}=\operatorname{bdiag}\left\{\boldsymbol{G}_{1}, \ldots, \boldsymbol{G}_{K}\right\}, \boldsymbol{H}=\left(\boldsymbol{h}_{1}, \ldots, \boldsymbol{h}_{K}\right), \boldsymbol{W}=\left(\boldsymbol{w}_{1}, \ldots, \boldsymbol{w}_{K}\right)$ as it us shown on fig. 1 .
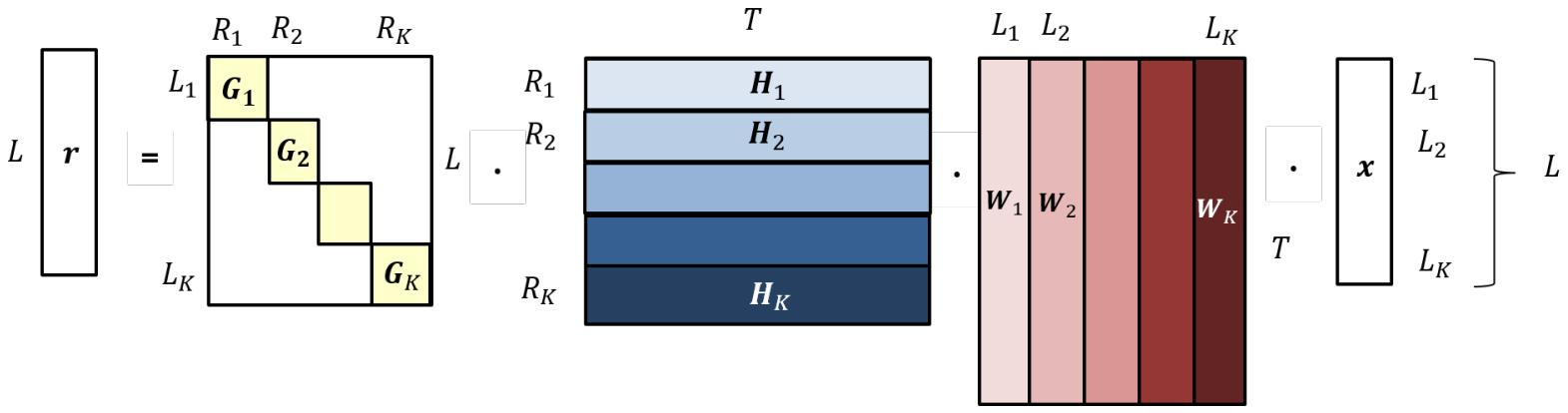

Figure 1: System model. Multi-User Precoding allows to transmit different information to different users simultaneously. 


\subsection{Singular Value Decomposition of the Channel}

It is convenient [14] to represent channel matrix of UE $k$ via its reduced Singular Value Decomposition (SVD) [14]:

$$
\boldsymbol{H}_{k}=\boldsymbol{U}_{k}^{H} \boldsymbol{S}_{k} \boldsymbol{V}_{k}, \quad \boldsymbol{U}_{k} \boldsymbol{U}_{k}^{H}=\boldsymbol{U}_{k}^{H} \boldsymbol{U}_{k}=\boldsymbol{I}_{R_{k}}, \quad \boldsymbol{S}_{k}=\operatorname{diag}\left\{s_{1}, \ldots, s_{R_{k}}\right\}, \quad \boldsymbol{V}_{k} \boldsymbol{V}_{k}^{H}=\boldsymbol{I}_{R_{k}} .
$$

Where the channel matrix for user $k, \boldsymbol{H}_{k} \in \mathbb{C}^{R_{k} \times T}$ contains channel vectors $\boldsymbol{h}_{i} \in \mathbb{C}^{T}$ by rows, the singular values $\boldsymbol{S}_{k} \in \mathbb{C}^{R_{k} \times R_{k}}$ are sorted by descending, $\boldsymbol{U}_{k} \in \mathbb{C}^{R_{k} \times R_{k}}$ is a unitary matrix of left singular vectors, and matrix $\boldsymbol{V}_{k} \in \mathbb{C}^{R_{k} \times T}$ consists of right singular vectors vector-rows Collecting all users together, we may write the following channel matrix decomposition.

Lemma 1. Denote $\boldsymbol{H}=\left(\boldsymbol{H}_{1}, \ldots, \boldsymbol{H}_{K}\right) \in \mathbb{C}^{T \times R}$ the contamination of individual channel vectors $\boldsymbol{H}_{k}$. Similarly, $\boldsymbol{U}=\operatorname{bdiag}\left\{\boldsymbol{U}_{1}, \ldots, \boldsymbol{U}_{K}\right\}, \boldsymbol{S}=\operatorname{bdiag}\left\{\boldsymbol{S}_{1}, \ldots, \boldsymbol{S}_{K}\right\}, \boldsymbol{V}=\left(\boldsymbol{V}_{1}, \ldots, \boldsymbol{V}_{K}\right)$, where $S V D$ for each $U E k$ is given by (2). The following representation holds

$$
\boldsymbol{H}=\boldsymbol{U}^{H} \boldsymbol{S} \boldsymbol{V}, \quad \boldsymbol{U} \boldsymbol{U}^{H}=\boldsymbol{U}^{H} \boldsymbol{U}=\boldsymbol{I}_{R}, \quad \boldsymbol{S}=\operatorname{diag}\left\{s_{1,1}, \ldots, s_{1, R_{1}}, \ldots, s_{K, R_{K}}\right\}, \quad \boldsymbol{C} \equiv \boldsymbol{V} \boldsymbol{V}^{H}-\boldsymbol{I}_{R} \neq 0 .
$$

Note that this representation is not actually SVD-decomposition of the matrix $\boldsymbol{H}$ : vectors $\boldsymbol{v}_{k, j}, \boldsymbol{v}_{l, i}$ that correspond to different UE $k \neq l$ are not generally orthogonal. Nonetheless, this representation has important properties that make it useful: the matrix $\boldsymbol{S}=\operatorname{diag}\left(\boldsymbol{S}_{k}\right) \in \mathbb{C}^{R \times R}$ is a diagonal matrix and $\boldsymbol{U}=\operatorname{bdiag}\left(\boldsymbol{U}_{k}\right) \in \mathbb{C}^{R \times R}$ is a block-diagonal unitary matrix. This allows UE to compensate factor $\boldsymbol{U} \boldsymbol{S}$ by the Detection (each UE deals with its own $\boldsymbol{U}_{k} \boldsymbol{S}_{k}$ ). Thus, on the transmitter side, it is sufficient to invert only the matrix $\boldsymbol{V}$, which in itself is much simpler than the channel $\boldsymbol{H}$ : first, its rows have a unit norm and, second, it is a natural object for Rank adaptation problem [20]. We will heavily use the Lemma (1) in the following.

\subsection{Conjugate Detection Matrix and the System}

In the previous section, we have introduced the notation of SVD (eq. 2). Usually the transmitter sends to UE several layers and the number of layers (rank) is less than the number of UE antennas $\left(L_{k} \leqslant R_{k}\right)$. In this case it is natural to choose for transmission the first $L_{k}$ vectors from $\boldsymbol{V}_{k}$ that correspond to the $L_{k}$ largest singular values from $\boldsymbol{S}_{k}$. Denote by $\widetilde{\boldsymbol{S}}_{k} \in \mathbb{C}^{L_{k} \times L_{k}}$ the first $L_{k}$ largest singular values from $\boldsymbol{S}_{k}$, and by $\widetilde{\boldsymbol{U}}_{k}^{H} \in \mathbb{C}^{R_{k} \times L_{k}}, \widetilde{\boldsymbol{V}}_{k} \in \mathbb{C}^{L_{k} \times T}$ the first $L_{k}$ left and right singular vectors that correspond to $\widetilde{\boldsymbol{S}}_{k}$ :

$$
\widetilde{\boldsymbol{S}}_{k}=\operatorname{diag}\left\{s_{k, 1}, \ldots, s_{k, L_{k}}\right\}, \quad \widetilde{\boldsymbol{U}}_{k}^{H}=\left[\boldsymbol{u}_{k, \cdot 1}^{H}, \ldots, \boldsymbol{u}_{k, L_{k}}^{H}\right], \quad \widetilde{\boldsymbol{V}}_{k}^{H}=\left[\boldsymbol{v}_{k, \cdot 1}^{H}, \ldots, \boldsymbol{v}_{k, L_{k}}^{H}\right],
$$

i.e. $\operatorname{rank} \widetilde{\boldsymbol{V}}_{k}=L_{k} \leq R_{k}=\operatorname{rank} \boldsymbol{V}_{k}$. Numbers $L_{k}$ (and maybe particular selection of $\widetilde{\boldsymbol{V}}_{k}$ ) are defined during the Rank Adaptation problem that, along with Scheduler, is solved before Precoding. For the Rank adaptation problem, we refer for example to [20] and in what follows we consider $L_{k}, \widetilde{\boldsymbol{V}}_{k}$ already chosen.

After Precoding and transmission, on the UE $k$ side, we have to choose the rectangular Detection $\boldsymbol{G}_{k} \in \mathbb{C}^{L_{k} \times R_{k}}$ that takes into account UE Rank $L_{k}$. The way UE performs Detection heavily affects the total performance and different Detection algorithms require different optimal Precodings. The best way would be to choose Precoding and Detection consistently but this is hardly possible due to the distributed nature of wireless communication. Nevertheless, there are ideas on how to adjust the Precoding matrix assuming a particular way of Detection on the UE side at the transmitter [21]. We do not consider such an approach in this paper, although it can be used to further improve our main proposal.

To conduct analytical calculations, we assume the Conjugate Detection (CD) in the following form:

$$
\boldsymbol{G}^{C}:=\widetilde{\boldsymbol{S}}^{-1} \widetilde{\boldsymbol{U}} \in \mathbb{C}^{L \times R}, \quad \boldsymbol{G}_{k}^{C}=\widetilde{\boldsymbol{S}}_{k}^{-1} \widetilde{\boldsymbol{U}}_{k} \in \mathbb{C}^{L_{k} \times R_{k}}
$$

Theorem 1. Assuming Conjugate Detection (5), symbols $\boldsymbol{x}$ of the system will flow only through the channel singular vectors $\widetilde{\boldsymbol{V}}$ as $\boldsymbol{r}=\widetilde{\boldsymbol{V}} \boldsymbol{W} \boldsymbol{x}+\widetilde{\boldsymbol{S}}^{-1} \widetilde{\boldsymbol{U}} \boldsymbol{n}$.

Proof. Using Lemma (1) we can write the following identities for the channel model (eq. 11) :

$$
\begin{gathered}
\boldsymbol{G}^{C} \boldsymbol{H}=\widetilde{\boldsymbol{S}}^{-1} \widetilde{\boldsymbol{U}} \boldsymbol{U}^{H} \boldsymbol{S} \boldsymbol{V}=\widetilde{\boldsymbol{S}}^{-1}[\boldsymbol{I} \mid \boldsymbol{O}] \boldsymbol{S} \boldsymbol{V}=\widetilde{\boldsymbol{S}}^{-1}\left[\begin{array}{c|c}
\widetilde{\boldsymbol{S}} & \boldsymbol{O} \\
\hline \boldsymbol{O} & \boldsymbol{O}
\end{array}\right] \boldsymbol{V}=\widetilde{\boldsymbol{S}}^{-1} \widetilde{\boldsymbol{S}} \widetilde{\boldsymbol{V}}=\widetilde{\boldsymbol{V}} \\
\boldsymbol{r}=\boldsymbol{G}^{C}(\boldsymbol{H} \boldsymbol{W} \boldsymbol{x}+\boldsymbol{n})=\widetilde{\boldsymbol{S}}^{-1} \widetilde{\boldsymbol{U}}(\boldsymbol{H} \boldsymbol{W} \boldsymbol{x}+\boldsymbol{n})=\widetilde{\boldsymbol{S}}^{-1} \widetilde{\boldsymbol{U}}\left(\boldsymbol{U}^{H} \boldsymbol{S} \boldsymbol{V} \boldsymbol{W} \boldsymbol{x}+\boldsymbol{n}\right)=\widetilde{\boldsymbol{V}} \boldsymbol{W} \boldsymbol{x}+\widetilde{\boldsymbol{S}}^{-1} \widetilde{\boldsymbol{U} n}
\end{gathered}
$$


Corollary 1. Transmitting data via vectors $\widetilde{\boldsymbol{V}}$ and assuming Conjugate Detection (5), the effective noise is

$$
\widetilde{\boldsymbol{S}}^{-1} \widetilde{\boldsymbol{U}} \boldsymbol{\sim} \sim \mathcal{C N}\left(0, \widetilde{\boldsymbol{S}}^{-2}\right)
$$

Remark 1. The Corollary 1 is essential for our study, since it gives the idea to use $\widetilde{\boldsymbol{S}}^{-2} \in \mathbb{C}^{L \times L}$ in regularization part of Precoding to take into account the correct effective noise $\widetilde{\boldsymbol{S}}^{-1} \widetilde{\boldsymbol{U}} \boldsymbol{n}$.

Corollary 2. One may notice that we have completely moved away from user antennas of shapes $R_{k}$ and $R$ in the equation (7) and work with user layers of shapes $L_{k}$ and $L$, where $L_{k} \leqslant R_{k} \forall k=1 \ldots K$.

Corollary 3. Finally, one may notice that it is sufficient to only perform Partial SVD of the channel $\boldsymbol{H}_{k} \in \mathbb{C}^{R_{k} \times T}$, keeping just the first $L_{k}$ singular values and vectors for each user $k$ such as

$$
\boldsymbol{H}_{k} \approx \widetilde{\boldsymbol{U}}_{k}^{H} \widetilde{\boldsymbol{S}}_{k} \widetilde{\boldsymbol{V}}_{k}
$$

Remark. Assuming the Corollaries (2,3), in what follows we will omit the tilde and write $\boldsymbol{U}_{k}, \boldsymbol{S}_{k}, \boldsymbol{V}_{k}$ instead of $\widetilde{\boldsymbol{U}}, \widetilde{\boldsymbol{S}}, \widetilde{\boldsymbol{V}}_{k}$ correspondingly.

We assume that the total channel $\boldsymbol{H}$, the number $K$ of UE and their ranks $L_{k}$ are known given values. This means that the Scheduler problem (which UE are to be served from the set of active UE) and Rank adaptation problem (which rank is provided to each UE) are already solved. This is usually the case in real networks. Scheduler and Rank adaptation problems are complicated and important radio resource management problems themselves but are out of the scope of this study (for examples of Scheduler problem we refer to [22] and bibliography within, for Rank adaptation - [20] and [21]. These algorithms affect the properties of matrix $\boldsymbol{H}$, e.g. Scheduler can choose only UE with small enough correlations $\left\|\boldsymbol{H} \boldsymbol{H}^{H}-\boldsymbol{I}\right\| \leqslant \varepsilon$. We take this into account by considering two scenarios: with random UE channels and UE channels with small correlations.

\subsection{Quality Measures}

\subsubsection{General Case}

The following functions are used to measure the quality of the Precoding methods. These functions are based not on the actual sending symbols $\boldsymbol{x} \in \mathbb{C}^{L}$, but some distribution of them [18]. Thus, we get the common function for all assumed symbols, which can be sent using the specified Precoding matrix.

The Signal-to-Interference-and-Noise functional of the $l=l(k)$-th symbol and user $k$ is defined as:

$$
\operatorname{SINR}_{l}\left(\boldsymbol{W}, \boldsymbol{H}_{k}, \boldsymbol{g}_{l}, \sigma, P\right):=\frac{\left|\boldsymbol{g}_{l} \boldsymbol{H}_{k} \boldsymbol{w}_{l}\right|^{2}}{\sum_{i \neq l}^{L}\left|\boldsymbol{g}_{l} \boldsymbol{H}_{k} \boldsymbol{w}_{i}\right|^{2}+\left\|\boldsymbol{g}_{l}\right\|^{2} \frac{\sigma^{2}}{P}} .
$$

The formula (10) shows the ratio between the useful and harmful parts of the signal. It depends on the whole Precoding matrix $\boldsymbol{W} \in \mathbb{C}^{M \times L}$, where the complex vector $\boldsymbol{w}_{l} \in \mathbb{C}^{M}$ denotes the Precoding for the $l$-th symbol, on the channel matrix $\boldsymbol{H}_{k} \in \mathbb{C}^{R_{k} \times M}$ of the $k$-th user, the Detection vector $\boldsymbol{g}_{l} \in \mathbb{C}^{R_{k}}$ of the $l$-th symbol, and global constants of the system noise $\sigma^{2}$ and the station power $P$. The formula 10 can be efficiently computed for all $L$ layers using several matrix multiplications and summations.

Usually an effective $\operatorname{SINR}$ is introduces $\operatorname{SINR}_{k}^{e f f}=f\left(\operatorname{SINR}_{1} \ldots S I N R_{L_{k}}\right)$ and there are different approaches to estimate it. Analysing results from (11), we see that simple geometrical mean can be a suitable model for theoretical study as it gives intermediate results between the realistic ones for QAM64 and QAM256 (see fig. 11). Denote $\mathcal{L}_{k}$ as the set of symbols for $k$-th user, then:

$$
\operatorname{SINR}_{k}^{e f f}\left(\boldsymbol{W}, \boldsymbol{H}_{k}, \boldsymbol{G}_{k}, \sigma, P\right):=\left(\prod_{l \in \mathcal{L}_{k}} \operatorname{SINR}_{l}\left(\boldsymbol{W}, \boldsymbol{H}_{k}, \boldsymbol{g}_{l}, \sigma, P\right)\right)^{\frac{1}{L_{k}}}
$$

This multi-criteria optimization can be decomposed into one-criterial optimization in different ways. We consider the sum of UE Spectral Efficiencies $\left(S E_{k}\right)$ over all UE. $S E_{k}$ refers to the information rate that can be transmitted over a given bandwidth for a certain UE. In the case of $R_{k}=1$ it is bounded by Shannon's theorem:

$$
S E_{k}\left(\boldsymbol{W}, \boldsymbol{S}_{k}, \boldsymbol{V}_{k}, \boldsymbol{n}\right):=L_{k} \log _{2}\left(1+\operatorname{SINR}_{k}^{\text {eff }}\left(\boldsymbol{W}, \boldsymbol{S}_{k}, \boldsymbol{V}_{k}, \boldsymbol{n}\right)\right) .
$$

This is theoretically supreme for the possible transmission Rate via $L_{k}$ resources, and recent modulation and coding schemes along with HARQ retransmission and BLER management allow achieving Rate very close to the Shannon entropy. 
In the case of several layers, generally speaking, $S E_{k}$ of $\mathrm{UE}_{k}$ is not a sum of $\log _{2}$ over its layers because there is one common transport block that is transmitted via all these layers, thus coding and modulation algorithms are also common.

Finally, Rates of UE are additive values and it is natural to consider the total $S E$ of the base station

$$
S E(\boldsymbol{W}, \boldsymbol{S}, \boldsymbol{V}, \boldsymbol{n})=\sum_{k=1}^{K} S E_{k}\left(\boldsymbol{W}, \boldsymbol{S}_{k}, \boldsymbol{V}_{k}, \boldsymbol{n}\right), \quad S E_{k}\left(\boldsymbol{W}, \boldsymbol{S}_{k}, \boldsymbol{V}_{k}, \boldsymbol{n}\right)=L_{k} \log _{2}\left(1+\operatorname{SINR}_{k}^{e f f}(\boldsymbol{W}, \boldsymbol{V}, \boldsymbol{n})\right)
$$

Note that SINR in formulas $(13)$ and $(11)$ is taken in linear values (not $\mathrm{dB}$ ).

There are a lot of other possible target functions, e.g. to improve the performance of cell edge UE (CEU). In [9] sec. 7] interested readers can find Pareto analysis of the multi-criteria statement and comparison of several target functions:

$$
S E_{\min }=\min _{k} S E_{k} \rightarrow \max \quad \text { or } S I N R_{\min }=\min _{1 \leqslant j \leqslant L} S I N R_{j} \rightarrow \max
$$

\subsubsection{Approximated Case}

Theorem 2. Assuming Conjugate Detection (5) and Theorem (1) we have come to the following approximation:

$$
\operatorname{SINR}_{l}\left(\boldsymbol{W}, \boldsymbol{H}_{k}, \boldsymbol{g}_{l}^{C}, \sigma^{2}, P\right)=\operatorname{SINR}_{l}^{C}\left(\boldsymbol{W}, \boldsymbol{v}_{l}, s_{l}, \sigma^{2}, P\right)=\frac{\left|\boldsymbol{v}_{l} \boldsymbol{w}_{l}\right|^{2}}{\sum_{i \neq l}^{L}\left|\boldsymbol{v}_{l} \boldsymbol{w}_{i}\right|^{2}+s_{l}^{-2} \frac{\sigma^{2}}{P}}
$$

Corollary 4. Spectral Efficiency function (13) can be approximate in the following way:

$$
\begin{aligned}
S E^{C}(\boldsymbol{W}, \boldsymbol{V}, \boldsymbol{S}, \sigma, P) & =\sum_{l=1}^{L} \log _{2}\left(1+\operatorname{SINR}_{l}^{C}\left(\boldsymbol{W}, \boldsymbol{v}_{l}, s_{l}, \sigma, P\right)\right)= \\
& \sum_{l=1}^{L} \log _{2}\left(\sum_{i=1}^{L}\left|\boldsymbol{v}_{l} \boldsymbol{w}_{i}\right|^{2}+s_{l}^{-2} \frac{\sigma^{2}}{P}\right)-\sum_{l=1}^{L} \log _{2}\left(\sum_{i \neq l}^{L}\left|\boldsymbol{v}_{l} \boldsymbol{w}_{i}\right|^{2}+s_{l}^{-2} \frac{\sigma^{2}}{P}\right)
\end{aligned}
$$

One may notice that we have completely moved away from user antennas of shapes $R_{k}$ and $R$ and work only with user layers of shapes $L_{k}$ and $L$. The formula now does not depend on channel matrix $\boldsymbol{H}_{k} \in \mathbb{C}^{R_{k} \times M}$ but on the eigenvectors of the $l$-th layer $\boldsymbol{v}_{l} \in \mathbb{C}^{M}$, which has length $M$ by the number of antennas. The inversed squared singular values $s_{l}^{-2} \in \mathbb{R}$ scale the noise, which is very essential in the new method construction.

Additionally, we consider the function of Single-User SINR for the $k$-th user:

$$
\operatorname{SUSINR}_{k}\left(\widetilde{\boldsymbol{S}}_{k}, \sigma, P\right):=\frac{P}{L_{k} \sigma^{2}}\left(\prod_{l \in \mathcal{L}_{k}} s_{l}^{2}\right)^{\frac{1}{L_{k}}}
$$

The formula 16 reflects the quality of the channel for the specified UE without taking into account other users. It depends on the greatest $L_{k}$ singular values $\widetilde{\boldsymbol{S}}_{k} \in \mathbb{R}^{L_{k} \times L_{k}}$ of the $k$-th user channel matrix $\boldsymbol{H}_{k} \in \mathbb{C}^{R_{k} \times T}$ and may be derived from the (10) and (11) formulas assuming Single-User case, MRT or ZF Precoding matrix and Conjugate Detection (5). We will use this function in our experiments as a universal channel characteristic including system noise $\sigma^{2}$ and station power $P$.

\subsection{Power Constraints}

Assume the total power of the system as $P$ and that the sent vector has unit norm $\mathbb{E}\{\|\boldsymbol{x}\|\}=1$. The total power constraints impose the following conditions on the Precoding matrix: $\|\boldsymbol{W}\|^{2} \leqslant P$. We can formulate the realistic [9] per-antenna power constraints and meet the real requirements of the system for each transmitting antenna. Since we have $T$ equal antennas, the power limitation applied to each of them is $\frac{P}{T}$. The antenna power can be described in terms of the row norms of the Precoding matrix: $\left\|\boldsymbol{w}^{m}\right\|^{2} \leqslant \frac{P}{T}, m=1 \ldots T$. It is clear that per-antenna constraints satisfy the total power of the system: $\sum_{m=1}^{T}\left\|\boldsymbol{w}^{m}\right\|^{2} \leqslant P$. We restrict the maximum power of antennas by multiplying the 
Precoding matrix by the scalar, which allows us to satisfy the power constraints and save the geometry and desired properties of the constructed Precoding:

$$
\mu=\frac{\sqrt{P}}{\sqrt{T} \max _{m}\left\{\left\|\boldsymbol{w}^{m}\right\|\right\}_{m=1}^{T}}>0
$$

Remark. In some cases, we will omit the normalization constant $\mu$ (17) for simplicity, where it is clear.

\section{Precoding Matrix Construction}

\subsection{Maximum Ratio Transmission (MRT)}

A Maximum Ratio Transmission Precoding algorithm takes just single-user weights $\boldsymbol{V}^{H}$ from the SVD-decomposition. That approach leads, as reflected in its name, to the maximization of single-user power ignoring the interference. The MRT approach is preferred in noisy systems where the noise power is higher than inter-user interference [15].

$$
\boldsymbol{W}_{M R T}=\mu \boldsymbol{V}^{H}
$$

Then (7) yields a set of interfering channels:

$$
\boldsymbol{r}=\boldsymbol{V} \boldsymbol{W} \boldsymbol{x}+\boldsymbol{S}^{-1} \boldsymbol{U} \boldsymbol{n}=\mu \boldsymbol{V} \boldsymbol{V}^{H} \boldsymbol{x}+\boldsymbol{S}^{-1} \boldsymbol{U} \boldsymbol{n}
$$

\subsection{Zero-Forcing (ZF)}

The next modification of the Precoding algorithm performs decorrelation of the symbols using the inverse correlation matrix of the channel vectors. Such Precoding construction sends the signal beams to the users without creating any interference between them. Different from the MRT method, the Zero-Forcing approach is preferred when the potential inter-user interference is higher than the noise power, and the Spectral Efficiency quality improves by eliminating this interference [15].

$$
\boldsymbol{W}_{Z F}=\mu \boldsymbol{V}^{\dagger}=\mu \boldsymbol{V}^{H}\left(\boldsymbol{V} \boldsymbol{V}^{H}\right)^{-1}
$$

The following receiver model is:

$$
\boldsymbol{r}=\boldsymbol{V} \boldsymbol{W} \boldsymbol{x}+\boldsymbol{S}^{-1} \boldsymbol{U} \boldsymbol{n}=\mu \boldsymbol{V} \boldsymbol{V}^{H}\left(\boldsymbol{V} \boldsymbol{V}^{H}\right)^{-1} \boldsymbol{x}+\boldsymbol{S}^{-1} \boldsymbol{U} \boldsymbol{n}=\mu \boldsymbol{x}+\boldsymbol{S}^{-1} \boldsymbol{U} \boldsymbol{n}
$$

Theorem 3. Assume the channel matrix has the form of $\boldsymbol{H}=\boldsymbol{U}^{H} \boldsymbol{S} \boldsymbol{V}$ (Lemma 1]) and denote $\boldsymbol{F}=\boldsymbol{U} \boldsymbol{H}=\boldsymbol{S} \boldsymbol{V}$. Then, the following relation for Zero-Forcing holds:

$$
\boldsymbol{W}_{Z F}(\boldsymbol{F}) \boldsymbol{S}=\boldsymbol{W}_{Z F}(\boldsymbol{V})
$$

Proof.

$$
\begin{aligned}
\boldsymbol{W}_{Z F}(\boldsymbol{F}) \boldsymbol{S}=\boldsymbol{F}^{H}\left(\boldsymbol{F} \boldsymbol{F}^{H}\right)^{-1} \boldsymbol{S}=\boldsymbol{V}^{H} \boldsymbol{S}\left(\boldsymbol{S} \boldsymbol{V} \boldsymbol{V}^{H} \boldsymbol{S}\right)^{-1} \boldsymbol{S}= \\
\quad \boldsymbol{V}^{H} \boldsymbol{S} \boldsymbol{S}^{-1}\left(\boldsymbol{V} \boldsymbol{V}^{H}\right)^{-1} \boldsymbol{S}^{-1} \boldsymbol{S}=\boldsymbol{V}^{H}\left(\boldsymbol{V} \boldsymbol{V}^{H}\right)^{-1}=\boldsymbol{W}_{Z F}(\boldsymbol{V})
\end{aligned}
$$

\subsection{Regularized Zero-Forcing (RZF)}

In the geometrical sense, in the ZF method (20), beams are sent not directly to the users but with some deviation, which actually reduces the useful signal. The following modification corrects the beams, which allows some inter-user interference and significantly increases the payload.

In the practical sense, in the ZF method 20, the channel right inversion may not exist or matrix $\boldsymbol{V} \boldsymbol{V}^{H}$ may be ill-conditioned, making ZF poorly perform. There are many practical solutions to this problem based on regularization.

$$
\boldsymbol{W}_{R Z F}(\boldsymbol{V})=\mu \boldsymbol{V}^{H}\left(\boldsymbol{V} \boldsymbol{V}^{H}+\lambda \boldsymbol{I}\right)^{-1}
$$


Regularized Zero-Forcing is the most common method in real practice, and therefore we use it as the main reference method. As the baseline, we use the analytical form of the regularization matrix using $\lambda=\frac{L \sigma^{2}}{P}$ [28].

This method cannot cancel all multi-user and multi-layer interference. It admits some interference to maximize single-user power. It is used as a trade-off between using MRT and ZF Precoding [18] balancing between maximizing the signal power and minimizing the interference leakage and thus we need appropriately manage them by optimizing the regularization parameter depending on the noise level.

The RZF method has the following asymptotic properties [15]: if $\sigma^{2} \rightarrow \infty$, it becomes equivalent to $\boldsymbol{W}_{M R T}=\mu \boldsymbol{V}^{H}$, which is optimal in low SINR cases. And if we set $\sigma^{2}=0$, the formula becomes equal to ZF Precoding: $\boldsymbol{W}_{Z F}=$ $\mu \boldsymbol{V}^{H}\left(\boldsymbol{V} \boldsymbol{V}^{H}\right)^{-1}$, which is optimal in high SINR cases.

The Precoding matrix based on the un-normalized channel [16] in the case when the number of sending symbols $L$ is less than the number of receiver antennas may be written in the following form of RZF:

$$
\boldsymbol{W}_{R Z F}(\boldsymbol{F})=\mu \boldsymbol{F}^{H}\left(\boldsymbol{F} \boldsymbol{F}^{H}+\lambda \boldsymbol{I}\right)^{-1}
$$

Where $\boldsymbol{F}=\boldsymbol{S} \boldsymbol{V}$ parameter and $\lambda=\frac{L \sigma^{2}}{P}[28]$ are chosen taking into account noise level $\sigma^{2}=E\left[\boldsymbol{n}^{H} \boldsymbol{n}\right]$. Actually, this method is equivalent to the an un-normalized channel matrix $\boldsymbol{H}$, which in our case is the matrix $\boldsymbol{F}$, obtained after splitting user channel into multiple streams.

The following theorem explains what functional is optimized to obtain the RZF method 24].

Theorem 4. Let us assume the channel decomposition $\boldsymbol{H}=\boldsymbol{U}^{H} \boldsymbol{S} \boldsymbol{V}$ from the Lemma 1 The Precoding $\boldsymbol{W}_{R Z F}(\boldsymbol{V})=$ $\boldsymbol{V}^{H}\left(\boldsymbol{V} \boldsymbol{V}^{H}+\lambda \boldsymbol{I}\right)^{-1}$, where $\lambda=\frac{L \sigma^{2}}{P}[28]$ is the solution of the following optimization problem:

$$
J(\boldsymbol{W})=\|\boldsymbol{V} \boldsymbol{W}-\boldsymbol{I}\|_{2}^{2}+\lambda\|\boldsymbol{W}\|_{2}^{2} \rightarrow \min _{\boldsymbol{W}}
$$

Proof. Calculating the gradient and equating it to zero, we get:

$$
\begin{gathered}
\nabla J(\boldsymbol{W})=2 \boldsymbol{V}^{H}(\boldsymbol{V} \boldsymbol{W}-\boldsymbol{I})+2 \lambda \boldsymbol{W}=0 \Leftrightarrow\left(\boldsymbol{V}^{H} \boldsymbol{V}+\lambda \boldsymbol{I}\right) \boldsymbol{W}=\boldsymbol{V}^{H} \\
\boldsymbol{W}=\left(\boldsymbol{V}^{H} \boldsymbol{V}+\lambda \boldsymbol{I}\right)^{-1} \boldsymbol{V}^{H}=\boldsymbol{V}^{H}\left(\boldsymbol{V} \boldsymbol{V}^{H}+\lambda \boldsymbol{I}\right)^{-1}
\end{gathered}
$$

The last identity may be proved using multiplication by the $\left(\boldsymbol{V} \boldsymbol{V}^{H}+\lambda \boldsymbol{I}\right)$ matrix from the right side of the identity and by the $\left(\boldsymbol{V}^{H} \boldsymbol{V}+\lambda \boldsymbol{I}\right)$ from the left side of it.

\subsection{Adaptive Regularized Zero-Forcing (ARZF)}

Proposed Algorithm. Taking into account effective noise from Corollary (1), we propose another algorithm that can be called Singular-value based Adaptive RZF (ARZF), where $\lambda=\frac{L \sigma^{2}}{P}[28]$ and matrices $\boldsymbol{V}$ and $\boldsymbol{S}$ obtained from the Lemma (1).

$$
\boldsymbol{W}_{A R Z F}(\boldsymbol{V})=\mu \boldsymbol{V}^{H}\left(\boldsymbol{V} \boldsymbol{V}^{H}+\lambda \boldsymbol{S}^{-2}\right)^{-1}
$$

It allows us to apply different regularizations for the different users and layers corresponding to their singular values that also include UE path loss. Small singular values correspond to the weak signal layers and if $s_{i}^{2}<\frac{\sigma^{2}}{P}$ should be regularized harder than others. It would be useless to completely orthogonalize this channel in this case, and it would be more correct to transmit via the conjugate channel as in MRT. On the other hand, if $s_{i}^{2} \gg \frac{\sigma^{2}}{P}$ regularisation for such layer vanishes and $\mathrm{ZF}$ orthogonalization is used.

The following theorem explains the relation between $\boldsymbol{W}_{R Z F}$ and $\boldsymbol{W}_{A R Z F}$ Precoding.

Theorem 5. Assume the channel matrix has the form of $\boldsymbol{H}=\boldsymbol{U}^{H} \boldsymbol{S} \boldsymbol{V}$ (Lemma 1) and denote $\boldsymbol{F}=\boldsymbol{U} \boldsymbol{H}=\boldsymbol{S} \boldsymbol{V}$. Then, the following relation for ARZF holds:

$$
\boldsymbol{W}_{R Z F}(\boldsymbol{F}) \boldsymbol{S}=\boldsymbol{W}_{A R Z F}(\boldsymbol{V})
$$


Proof.

$$
\begin{aligned}
\boldsymbol{W}_{R Z F}(\boldsymbol{F}) \boldsymbol{S} & \equiv \boldsymbol{F}^{H}\left(\boldsymbol{F} \boldsymbol{F}^{H}+\lambda \boldsymbol{I}\right)^{-1} \boldsymbol{S}=\boldsymbol{V}^{H} \boldsymbol{S}\left(\boldsymbol{S} \boldsymbol{V} \boldsymbol{V}^{H} \boldsymbol{S}+\lambda \boldsymbol{I}\right)^{-1} \boldsymbol{S}= \\
& =\boldsymbol{V}^{H} \boldsymbol{S} \boldsymbol{S}^{-1}\left(\boldsymbol{V} \boldsymbol{V}^{H}+\boldsymbol{S}^{-1} \lambda \boldsymbol{I} \boldsymbol{S}^{-1}\right)^{-1} \boldsymbol{S}^{-1} \boldsymbol{S}=\boldsymbol{V}^{H}\left(\boldsymbol{V} \boldsymbol{V}^{H}+\lambda \boldsymbol{S}^{-2}\right)^{-1} \equiv \boldsymbol{W}_{A R Z F}(\boldsymbol{V})
\end{aligned}
$$

Right factor $\boldsymbol{S}$ in the r.h.s. of (29) can be interpreted as a special type of Power Allocation algorithm (see an interesting study in [9, sec. 7]) that distribute the total transmision power between layers.

Idea. We discovered ARZF formula (28) using the proper PCA-decomposition, which is stated in the Theorem (5).

In practice, it is better to use $\boldsymbol{W}_{R Z F}(\boldsymbol{V})$ rather than $\boldsymbol{W}_{R Z F}(\boldsymbol{F})$, because the norms of the rows of the matrix $\boldsymbol{F} \boldsymbol{F}^{H}+\lambda \boldsymbol{I}$ can differ sufficiently (by several orders!), which leads to unbalanced power distribution between layers (as state Theorem 5 another way is to apply a proper Power allocation for $\boldsymbol{W}_{R Z F}(\boldsymbol{F})$. On the other hand, the regularisation parameter of $\boldsymbol{W}_{R Z F}(\boldsymbol{F})$ is more natural and correct. The proposed $\boldsymbol{W}_{A R Z F}(\boldsymbol{V})$ combines the benefits from these two approaches and provides an envelope of them.

The following theorem explains what functional is optimized to obtain the proposal solution 28].

Theorem 6. Let us assume the channel decomposition $\boldsymbol{H}=\boldsymbol{U}^{H} \boldsymbol{S} \boldsymbol{V}$ from the Lemma 1 . The proposed Precoding $\boldsymbol{W}_{A R Z F}(\boldsymbol{V})=\boldsymbol{V}^{H}\left(\boldsymbol{V} \boldsymbol{V}^{H}+\lambda \boldsymbol{S}^{-2}\right)^{-1}$, where $\lambda=\frac{L \sigma^{2}}{P}[28]$, is the solution of the following optimization problem:

$$
J(\boldsymbol{W})=\|\boldsymbol{V} \boldsymbol{W}-\boldsymbol{I}\|_{\boldsymbol{S}}^{2}+\lambda\|\boldsymbol{W}\|_{I}^{2}=\|\boldsymbol{S}(\boldsymbol{V} \boldsymbol{W}-\boldsymbol{I})\|_{2}^{2}+\lambda\|\boldsymbol{W}\|_{2}^{2} \rightarrow \min _{\boldsymbol{W}}
$$

Proof. Calculating the gradient and equating it to zero, we get:

$$
\begin{gathered}
\nabla J(\boldsymbol{W})=2 \boldsymbol{V}^{H} \boldsymbol{S}(\boldsymbol{S} \boldsymbol{V} \boldsymbol{W}-\boldsymbol{S})+2 \lambda \boldsymbol{W}=0 \Leftrightarrow\left(\boldsymbol{V}^{H} \boldsymbol{S}^{2} \boldsymbol{V}+\lambda \boldsymbol{I}\right) \boldsymbol{W}=\boldsymbol{V}^{H} \boldsymbol{S}^{2} \\
\boldsymbol{W}=\left(\boldsymbol{V}^{H} \boldsymbol{S}^{2} \boldsymbol{V}+\lambda \boldsymbol{I}\right)^{-1} \boldsymbol{V}^{H} \boldsymbol{S}^{2}=\boldsymbol{V}^{H}\left(\boldsymbol{V} \boldsymbol{V}^{H}+\lambda \boldsymbol{S}^{-2}\right)^{-1}
\end{gathered}
$$

The last identity may be proved using multiplication by the $\left(\boldsymbol{V} \boldsymbol{V}^{H}+\lambda \boldsymbol{S}^{-2}\right)$ matrix from the right side of the identity and by the $\left(\boldsymbol{V}^{H} \boldsymbol{S}^{2} \boldsymbol{V}+\lambda \boldsymbol{I}\right)$ from the left side of it.

The meaning of the function $J(\boldsymbol{W}) \sqrt{31}$ is as follows. The second term $\lambda\|\boldsymbol{W}\|_{2}^{2}$ is the standard noise regularization part. And the first term, the norm $\|\boldsymbol{S}(\boldsymbol{V} \boldsymbol{W}-\boldsymbol{I})\|_{2}^{2}$, weighted by the matrix $\boldsymbol{S}$, weights more for the layers with higher singular values. And, therefore, the function is optimized more precisely for the layers with a higher signal quality compared to the layers with lower signal quality. In other words, Precoding vectors for layers with higher singular values become similar to Zero-Forcing Precoding, and for layers with lower singular values become similar to Maximum-Ratio Transmission, i.e. ARZF provides adaptive regularization. In the next section, we will see that this approach leads to a uniform increase in spectral efficiency compared to the basic method with unit weights.

\section{Numerical results}

We are testing the proposed approach in several scenarios generated using Quadriga [25] - open-source software for generating realistic radio channel impulse response. The main scenarios are 1) Rural Line-of-Sight 3GPP_38.901_RMa_LOS, and 2) Urban Non-Line-of-Sight 3GPP_38.901_RMa_NLOS [27]. For each scenario we generate 10 different channel matrices $H \in \mathbb{C}^{n_{\text {users }} \times 4 \times 64}$. The carrier frequency for each channel matrix is selected randomly over the bandwidth. User selection is described in the next section. The base station antenna array forms a grid with 8 placeholders along the $y$ axis and 4 placeholders along the $y$ axis. The receiver antenna array consists of two placeholders along the $x$ axis. Each placeholder contains two cross-polarized antennas. An interested reader can find detailed hyperparameters for antenna models and generation processes in the table (3. see Appendix). All unlisted Quadriga parameters are those set by default. 
A PREPRINT - JULY 1, 2021

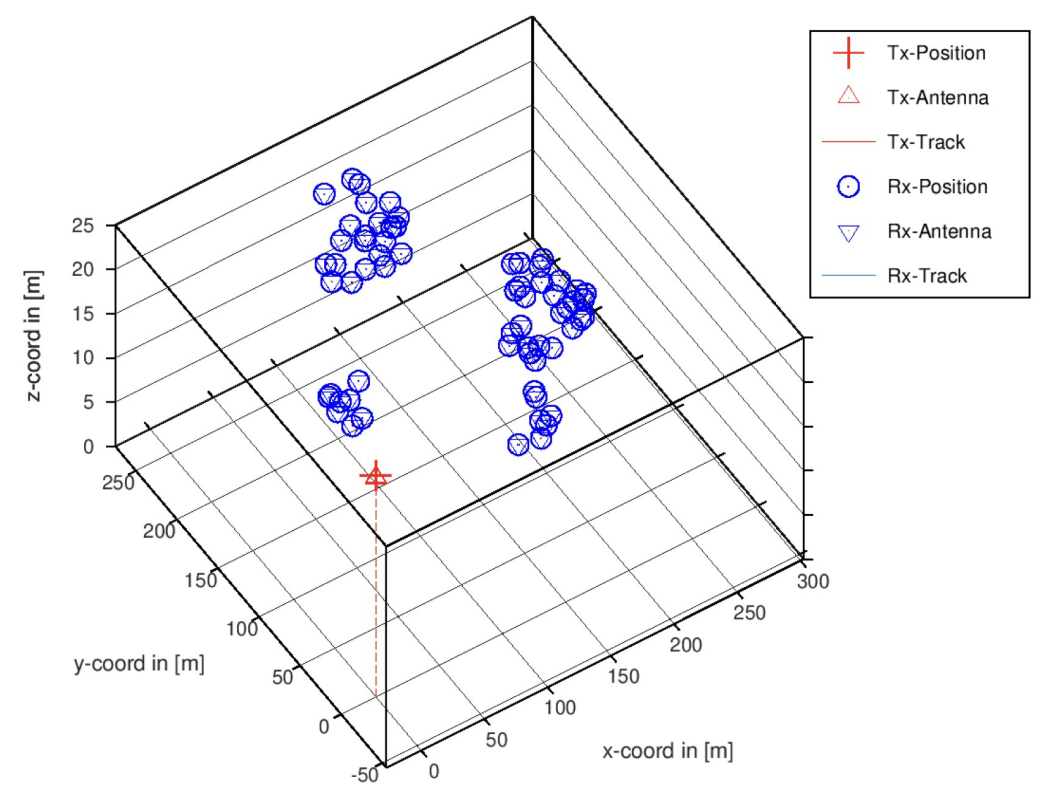

Figure 2: Example of the random generation of users for Urban setup. There are two buildings, and the users are assigned to either a cluster in a building, or to the ground near the building.

\subsection{Selecting user positions}

For the Rural LOS scenario, we model users in the suburban landscape. We locate users on the ground, set the height to $1.5 \mathrm{~m}$, and generate $x, y$ positions uniformly in the $120^{\circ}$ ray within $250 \mathrm{~m}$ from the base station. For the NLOS scenario, we locate users in the urban landscape. Firstly, we sample up to 8 cluster centres $x_{c}, y_{c}$ in the $120^{\circ}$ ray from the base station within $2000 \mathrm{~m}$ from the base station. Each cluster represents a part of a city building. We assign a random cluster height $z_{c}=1.5 \mathrm{~m}+(3 \cdot U(\{1, \ldots, 10\})-1)$, selecting the cluster floor in a building from the uniform distribution $U$. Secondly, for each user, we assign a cluster id $c(u)$ and sample $x_{u}, y_{u}$ position randomly over the $60 \mathrm{~m}$ circle around the cluster centre. Thirdly, we sample the height of the user, given the height of the cluster, $80 \%$ of users we place at the floors near the cluster floor $z_{u}=z_{c(u)}+3 \cdot U(\{-1,0,1\})$ m., and $20 \%$ of users we place outdoor $z_{u}=1.5 \mathrm{~m}$.

After generating channel matrices for a fairly large number of users (64), we select a subset of users, which are not too correlated, since too correlated users can be suited at a different time or frequency intervals. The correlation between users $i, j$ is measured as squared cosine between the main singular vector: $\cos ^{2}\left(v_{i}, v_{j}\right)=\left|\widetilde{\boldsymbol{V}}_{i 0}^{H} \widetilde{\boldsymbol{V}}_{j 0}\right|^{2}$.

Our user generation algorithm produces realistic setups for Urban case and is simple to implement and use in the following studies.

\section{Conclusion}

Multi-user Precoding optimization is a key problem in modern cellular wireless systems, which are based on massive MIMO technology. In this work, we propose sub-optimal analytical solutions of the Precoding optimization problem in a generalized mean squared error minimization (MMSE) framework, which leads to a sufficient increase in quality with the same computation time as in the baselines.

All investigated algorithms were studied in massive experiments using Quadriga [25] - open-source software for generating realistic radio channel impulse response. Our method shows monotonic improvement over heuristic methods with reasonable computation time. 


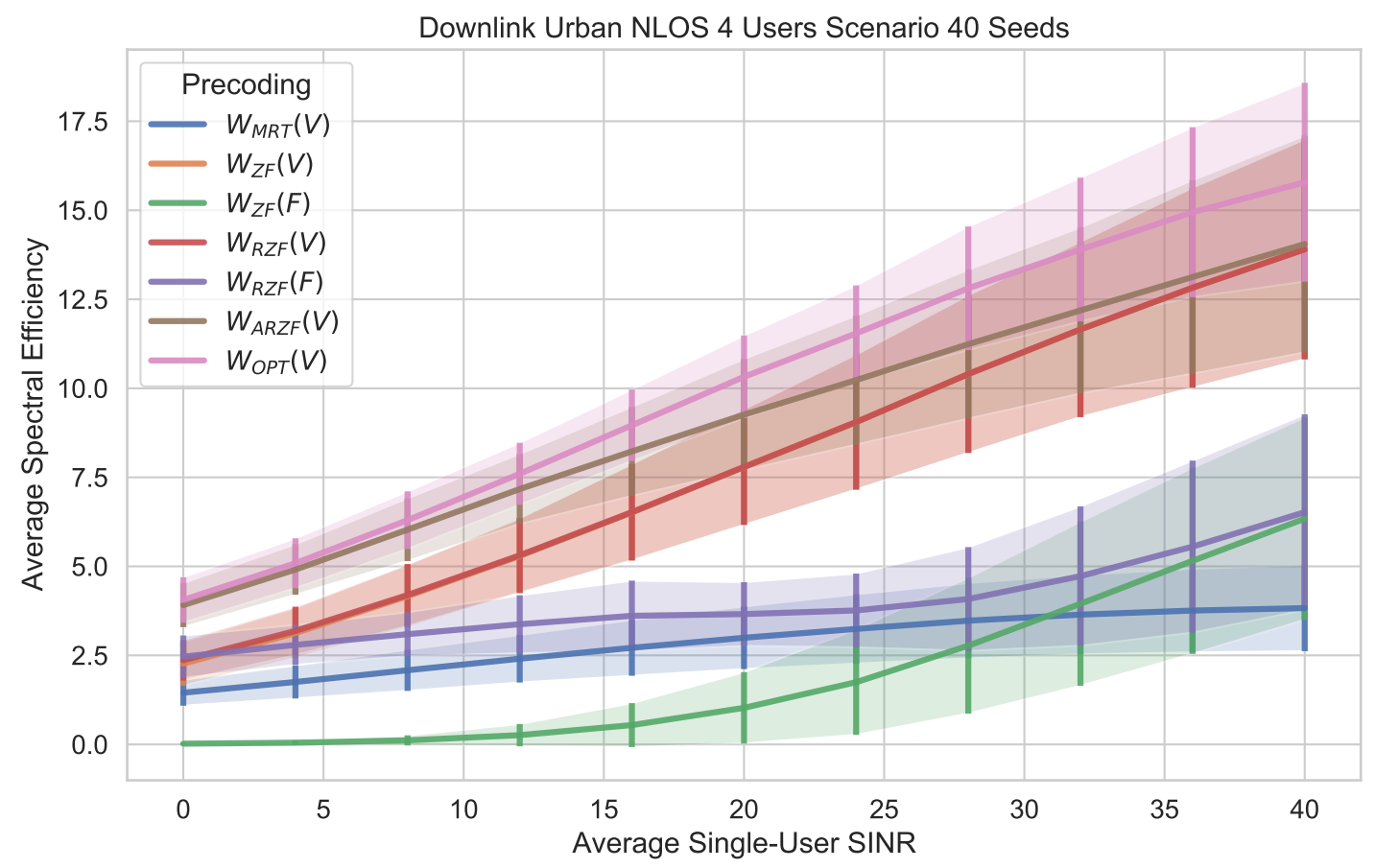

Figure 3: The graphs show Spectral Efficiency of the different Precoding algorithms in the Urban NLOS scenarios using path-losses. The main setting includes $K=4$ users, $T=64$ base station antennas, $R=16$ total number of receive antennas assuming $R_{k}=4$ antennas for each user, $L=8$ total number of transmitted symbols assuming $L_{k}=2$ symbols for each user.

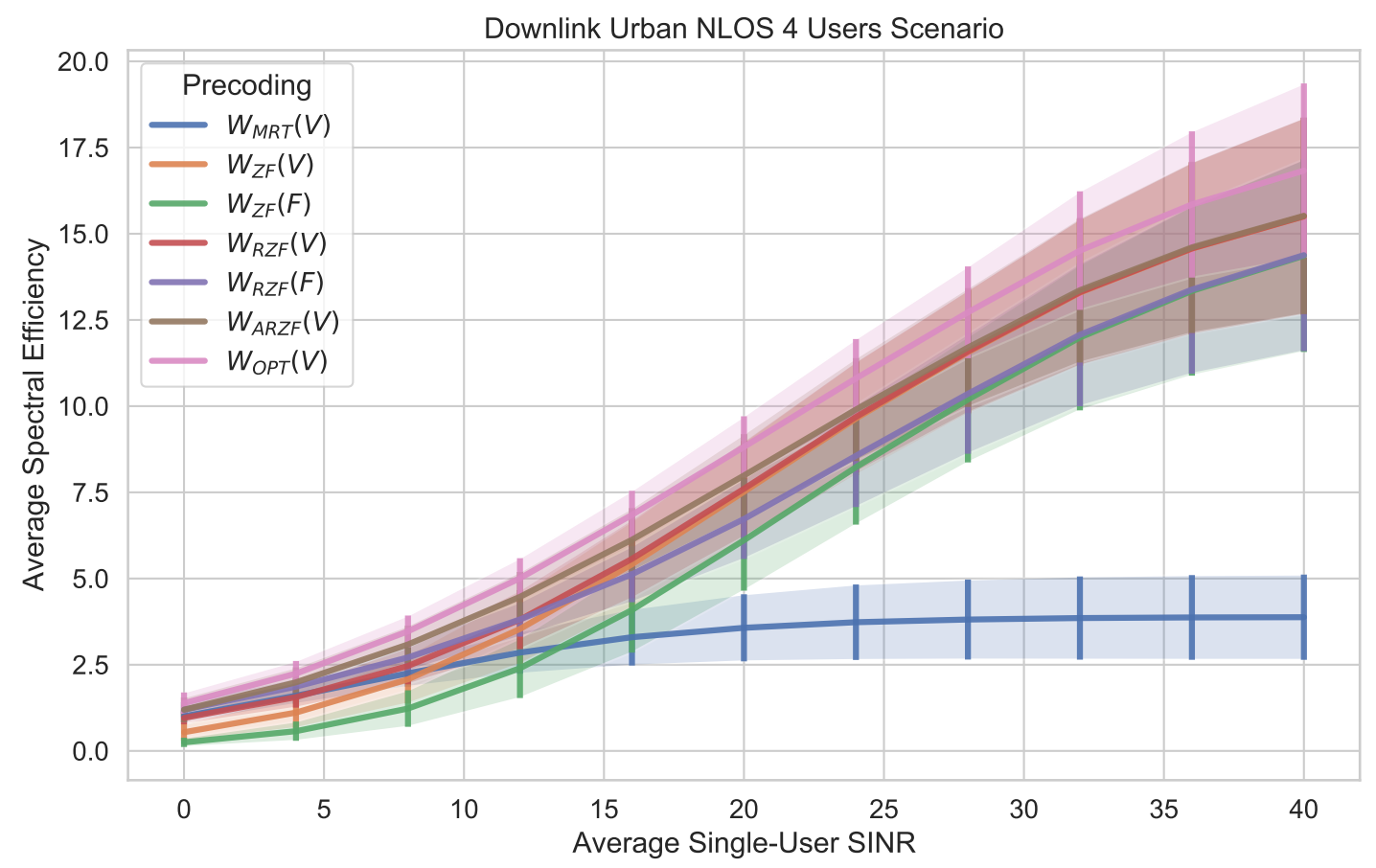

Figure 4: The graphs show Spectral Efficiency of the different Precoding algorithms in the Urban NLOS scenario. The main setting includes $K=4$ users, $T=64$ base station antennas, $R=16$ total number of receive antennas assuming $R_{k}=4$ antennas for each user, $L=8$ total number of transmitted symbols assuming $L_{k}=2$ symbols for each user. 


\begin{tabular}{|c|c|c|c|c|c|c|c|}
\hline $\begin{array}{l}\text { Precoding } \\
\text { SUSINR }\end{array}$ & $\boldsymbol{W}_{M R T}(\boldsymbol{V})$ & $\boldsymbol{W}_{Z F}(\boldsymbol{V})$ & $\boldsymbol{W}_{Z F}(\boldsymbol{F})$ & $\boldsymbol{W}_{R Z F}(\boldsymbol{V})$ & $\boldsymbol{W}_{R Z F}(\boldsymbol{F})$ & $\boldsymbol{W}_{A R Z F}(\boldsymbol{V})$ & $\boldsymbol{W}_{O P T}(\boldsymbol{V})$ \\
\hline 0 & 1.45 & 2.27 & 0.02 & 2.37 & 2.46 & 3.91 & 4.06 \\
\hline 4 & 1.75 & 3.13 & 0.05 & 3.19 & 2.79 & 4.91 & 5.09 \\
\hline 8 & 2.08 & 4.16 & 0.11 & 4.19 & 3.10 & 6.03 & 6.30 \\
\hline 12 & 2.41 & 5.29 & 0.26 & 5.31 & 3.37 & 7.17 & 7.60 \\
\hline 16 & 2.71 & 6.51 & 0.54 & 6.52 & 3.61 & 8.23 & 8.96 \\
\hline 20 & 2.99 & 7.79 & 1.03 & 7.79 & 3.66 & 9.26 & 10.32 \\
\hline 24 & 3.24 & 9.05 & 1.75 & 9.05 & 3.76 & 10.23 & 11.54 \\
\hline 28 & 3.47 & 10.40 & 2.77 & 10.40 & 4.08 & 11.24 & 12.81 \\
\hline 32 & 3.65 & 11.65 & 3.95 & 11.65 & 4.73 & 12.19 & 13.90 \\
\hline 36 & 3.76 & 12.83 & 5.16 & 12.83 & 5.55 & 13.13 & 14.95 \\
\hline 40 & 3.83 & 13.90 & 6.34 & 13.90 & 6.52 & 14.05 & 15.79 \\
\hline
\end{tabular}

Table 1: The table shows Spectral Efficiency of the different Precoding algorithms in the Urban NLOS scenario using path-losses. Proposal algorithm is $\boldsymbol{W}_{A R Z F}(\boldsymbol{V})$. Optimal regularization $\boldsymbol{W}_{O P T}(V)$ was configured using L-BFGS-B algorithm on the. The main setting includes $K=4$ users, $T=64$ base station antennas, $R=16$ total number of receive antennas assuming $R_{k}=4$ antennas for each user, $L=8$ total number of transmitted symbols assuming $L_{k}=2$ symbols for each user.

\begin{tabular}{|c|c|c|c|c|c|c|c|}
\hline $\begin{array}{l}\text { Precoding } \\
\text { SUSINR }\end{array}$ & $\boldsymbol{W}_{M R T}(\boldsymbol{V})$ & $\boldsymbol{W}_{Z F}(\boldsymbol{V})$ & $\overline{\boldsymbol{W}_{Z F}(\boldsymbol{F})}$ & $\boldsymbol{W}_{R Z F}(\boldsymbol{V})$ & $\boldsymbol{W}_{R Z F}(\boldsymbol{F})$ & $\boldsymbol{W}_{A R Z F}(\boldsymbol{V})$ & $\overline{\boldsymbol{W}_{O P T}(\boldsymbol{V})}$ \\
\hline 0 & 1.00 & 0.54 & 0.25 & 0.96 & 1.19 & 1.19 & 1.39 \\
\hline 4 & 1.60 & 1.11 & 0.57 & 1.56 & 1.86 & 1.99 & 2.24 \\
\hline 8 & 2.25 & 2.08 & 1.23 & 2.46 & 2.71 & 3.09 & 3.47 \\
\hline 12 & 2.8 & 3.54 & 2.40 & 3.81 & 3.81 & 4.47 & 5.01 \\
\hline 16 & 3.3 & 5.41 & 4.09 & 5.57 & 5.12 & 6.12 & 6.85 \\
\hline 20 & 3.57 & 7.52 & 6.11 & 7.60 & 6.73 & 7.99 & 8.82 \\
\hline 24 & 3.73 & 9.64 & 8.23 & 9.69 & 8.56 & 9.90 & 10.80 \\
\hline 28 & 3.81 & 11.56 & 10.19 & 11.59 & 10.36 & 11.70 & 12.72 \\
\hline 32 & 3.85 & 13.29 & 11.99 & 13.31 & 12.07 & 13.36 & 14.51 \\
\hline 36 & 3.87 & 14.57 & 13.34 & 14.58 & 13.38 & 14.60 & 15.85 \\
\hline 40 & 3.88 & 15.50 & 14.36 & 15.51 & 14.38 & 15.52 & 16.83 \\
\hline
\end{tabular}

Table 2: The table shows Spectral Efficiency of the different Precoding algorithms in the Urban NLOS scenario. The main setting includes $K=4$ users, $T=64$ base station antennas, $R=16$ total number of receive antennas assuming $R_{k}=4$ antennas for each user, $L=8$ total number of transmitted symbols assuming $L_{k}=2$ symbols for each user.

\section{References}

[1] Ngo, Hien Quoc, Erik G. Larsson, and Thomas L. Marzetta. "Energy and spectral efficiency of very large multiuser MIMO systems." IEEE Transactions on Communications 61.4 (2013): 1436-1449.

[2] Andrews, Jeffrey G., et al. "What will 5G be?." IEEE Journal on selected areas in communications 32.6 (2014): 1065-1082.

[3] Jaeckel, S., Raschkowski, L., Börner, K., \& Thiele, L. (2014). QuaDRiGa: A 3-D multi-cell channel model with time evolution for enabling virtual field trials. IEEE Transactions on Antennas and Propagation, 62(6), 3242-3256.

[4] Tran, Le-Nam, et al. "Beamformer designs for MISO broadcast channels with zero-forcing dirty paper coding." IEEE transactions on wireless communications 12.3 (2013): 1173-1185.

[5] Zheng, Kan, et al. "Survey of large-scale MIMO systems." IEEE Communications Surveys \& Tutorials 17.3 (2015): 1738-1760.

[6] Fatema, Nusrat, et al. "Massive MIMO linear Precoding: A survey." IEEE systems journal 12.4 (2017): $3920-3931$.

[7] Hoydis, Jakob, Stephan Ten Brink, and Mérouane Debbah. "Massive MIMO in the UL/DL of cellular networks: How many antennas do we need?." IEEE Journal on Selected Areas in Communications 31.2 (2013): 160-171. s 
A PREPRINT - JULY 1, 2021

\begin{tabular}{|c|c|}
\hline Parameter & Value \\
\hline \multicolumn{2}{|l|}{ Base station parameters } \\
\hline number of base stations & 1 \\
\hline position, $\mathrm{m}$ : $(\mathrm{x}, \mathrm{y}, \mathrm{z})$ axes & $(0,0,25)$ \\
\hline number of antenna placeholders (y axis) & 8 \\
\hline number of antenna placeholders ( $\mathrm{z}$ axis) & 4 \\
\hline distance between placeholders ( $y$ axis) & 0.5 wavelength \\
\hline distance between placeholders ( $z$ axis) & 1.7 wavelength \\
\hline antenna model & 3gpp-macro \\
\hline half-Power in azimuth direction, deg & 60 \\
\hline half-Power in elevation direction, deg & 10 \\
\hline front-to back ratio, $\mathrm{dB}$ & 20 \\
\hline total number of antennas & 64 \\
\hline \multicolumn{2}{|l|}{ Receiver parameters } \\
\hline number of placeholders at the receiver ( $x$ axis) & 2 \\
\hline distance between placeholders ( $x$ axis) & 0.5 wavelength \\
\hline antenna model & half-wave-dipole \\
\hline total number of antennas & 4 \\
\hline \multicolumn{2}{|l|}{ Quadriga simulation parameters } \\
\hline central band frequency & $3.5 \mathrm{GHz}$ \\
\hline 1 sample per meter (default value) & 1 \\
\hline include delay of the LOS path & 1 \\
\hline disable spherical waves (use_3GPP_baseline) & 1 \\
\hline \multicolumn{2}{|l|}{ Quadriga channel builders parameters } \\
\hline shadow fading sigma & 0 \\
\hline cluster splitting & False \\
\hline bandwidth & $100 \mathrm{MHz}$ \\
\hline number of subcarriers & 42 \\
\hline
\end{tabular}

Table 3: Quadriga generation parameters

[8] Björnson, Emil, Eduard Jorswieck, and Bjorn Ottersten. "Impact of spatial correlation and Precoding design in OSTBC MIMO systems." IEEE Transactions on Wireless Communications 9.11 (2010): 3578-3589.

[9] Björnson, Emil, Jakob Hoydis, and Luca Sanguinetti. "Massive MIMO Networks: Spectral, energy, and hardware efficiency." Foundations and Trends in Signal Processing 11.3-4 (2017): 154-655.

[10] A. Aitken, 1936, IV.-On Least Squares and Linear Combination of Observations, Proceedings of the Royal Society of Edinburgh, 55 42-48 doi:10.1017/S0370164600014346

[11] D. Tse, P. Viswanath, 2005, Fundamentals of Wireless Communication. Cambridge: Cambridge University Press. doi:10.1017/CBO9780511807213

[12] A. Zaidi et. al., 5G Physical Layer Principles, Models and Technology Components, 2018, Academic Press. ISBN: 978-0-12-814578-4

[13] Zhu, 2006, Tomlinson-Harashima Precoding https://arxiv.org/abs/1809.10076v1

Karlsson Bjornson Local Partial Zero-Forcing Precoding for Cell-Free Massive MIMO

[14] Sun, Liang, and Matthew R. McKay. "Eigen-based transceivers for the MIMO broadcast channel with semiorthogonal user selection." IEEE Transactions on Signal Processing 58.10 (2010): 5246-5261.

[15] Parfait, Tebe, Yujun Kuang, and Kponyo Jerry. "Performance analysis and comparison of ZF and MRT based downlink massive MIMO systems." 2014 Sixth International Conference on Ubiquitous and Future Networks (ICUFN). IEEE, 2014.

[16] Nguyen, Long D., et al. "Multi-user Regularized Zero-Forcing Beamforming." IEEE Transactions on Signal Processing 67.11 (2019): 2839-2853.

[17] Dhakal, S. (2019). High rate signal processing schemes for correlated channels in 5G networks.

[18] Björnson, E., Bengtsson, M., Ottersten, B. (2014). Optimal multiuser transmit beamforming: A difficult problem with a simple solution structure [lecture notes]. IEEE Signal Processing Magazine, 31(4), 142-148. 
[19] Suzuki, Takao. "L1 generalized inverse beam-forming algorithm resolving coherent/incoherent, distributed and multipole sources." Journal of Sound and Vibration 330.24 (2011): 5835-5851.

[20] N. H. Mahmood, G. Berardinelli, F. M. L. Tavares, M. Lauridsen, P. Mogensen and K. Pajukoski, "An Efficient Rank Adaptation Algorithm for Cellular MIMO Systems with IRC Receivers," 2014 IEEE 79th Vehicular Technology Conference (VTC Spring), 2014, pp. 1-5, DOI: 10.1109/VTCSpring.2014.7022820.

[21] S. Shi et. al., "Downlink MMSE Transceiver Optimization for Multiuser MIMO Systems: Duality and SumMSE Minimization," in IEEE Transactions on Signal Processing, vol. 55, no. 11, pp. 54365446, Nov. 2007.

[22] L. Tran, "An Iterative Precoder Design for Successive ZeroForcing Precoded Systems," in IEEE Communications Letters, vol. 16, no. 1, pp. 1618, January 2012.

[23] T. E. Bogale and L. Vandendorpe, "Sum MSE optimization for downlink multiuser MIMO systems with per antenna power constraint: Downlink-uplink duality approach," 2011 IEEE 22nd International Symposium on Personal, Indoor and Mobile Radio Communications, Toronto, ON, 2011, pp. 2035-2039, DOI: 10.1109/PIMRC.2011.6139871.

[24] Wiesel, Ami, Yonina C. Eldar, and Shlomo Shamai. "Zero-forcing Precoding and generalized inverses." IEEE Transactions on Signal Processing 56.9 (2008): 4409-4418.

[25] Jaeckel, S., Raschkowski, L., Börner, K., \& Thiele, L. (2014). QuaDRiGa: A 3-D multi-cell channel model with time evolution for enabling virtual field trials. IEEE Transactions on Antennas and Propagation, 62(6), 3242-3256.

[26] Ren, Bin, et al. "Low-complexity MMSE-IRC algorithm for uplink massive MIMO systems." Electronics Letters 53.14 (2017): 972-974.

[27] Bohagen, F., Orten, P., \& Oien, G. E. (2005, March). Construction and capacity analysis of high-rank line-of-sight MIMO channels. In IEEE Wireless Communications and Networking Conference, 2005 (Vol. 1, pp. 432-437). IEEE.

[28] Peel, Christian B., Bertrand M. Hochwald, and A. Lee Swindlehurst. "A vector-perturbation technique for nearcapacity multiantenna multiuser communication-part I: channel inversion and regularization." IEEE Transactions on Communications 53.1 (2005): 195-202. 